\title{
A Rhodopsin-Like Ionic Gate Fabricated with Graphene Oxide and Isomeric DNA Switch for Efficient Photocontrol of Ion Transport
}

Liu Shi,${ }^{\dagger}$ Chaoli Mu,${ }^{\dagger}$ Tao Gao,${ }^{\S}$ Wenxin Chai,${ }^{\dagger}$ Anzhi Sheng, $₫$ Tianshu Chen,${ }^{\ddagger}$ Jie Yang, ${ }^{\dagger}$ Xiaoli Zhu, ${ }^{*}+$ and Genxi $\mathrm{Li}^{*}, \dagger,+$

†State Key Laboratory of Pharmaceutical Biotechnology, School of Life Sciences, Nanjing University, Nanjing 210023, P. R. China

$\S$ Jiangsu Key Laboratory for Molecular and Medical Biotechnology, College of Life Sciences, Nanjing Normal University, Nanjing 210023, P. R. China

¿Center for Molecular Recognition and Biosensing, School of Life Sciences, Shanghai University, Shanghai 200444, P. R. China 


\section{Experiment Section}

Materials and Reagents. Azo-incorporated DNA oligonucleotides, 5'- $\mathrm{CHO}-\left(\mathrm{CH}_{2}\right)_{6}-$ CCTAGCAACAGACCGCACTTTATGATAGCAA(Azo)GC(Azo)TA(Azo)GG-3', were ordered from Sangon Biotech Co., Ltd (Shanghai, China). PAA was purchased from Hefei Puyuan Nanotechnology Co., Ltd (Anhui, China). Single layer graphene oxide was purchased from Nanjing XFNano Material Tech Co., Ltd (Nanjing, China). (3-aminopropyl)triethoxysilane (APTES), potassium chloride $(\mathrm{KCl})$, and other reagents of certified analytical grade were obtained from Sigma-Aldrich (Shanghai, China) or from Sigma-Aldrich Co., Ltd (St Louis, MO, USA). All solutions were prepared with deionized water, which was purified with a Milli-Q purification system (Bedford, MA, USA) to a resistance of $18.2 \mathrm{M} \Omega \mathrm{cm}$. Human serum samples were supplied by the Second Hospital of Nanjing and approved by the medical ethics committee of the hospital.

Instrumentation. The electrochemical detection was performed in an H-type electrolytic cell made of quartz on an electrochemical workstation (CHI 660D, Chenhua, China). The morphologies of the prepared PAA channel were characterized using a scanning electron microscope (SEM, SU8020, Japan). The bare barrier layer of PAA and GO-combined barrier layer were observed using an atomic force microscopy (AFM, Bruker, Germany). The modification process was characterized by X-ray photoelectron spectroscopy (XPS, ESCALAB 250XI, Thermo Fisher Scientific, U.S.A.), as shown in Table S1-4. The photoresponsive property of Azo-DNA was investigated by UV-2450 UV-VIS spectrophotometer (UV-2450, SHIMADZU, England). Electrochemical measurements were performed on a CHI660D electrochemical workstation $(\mathrm{CH}$ Instruments, Austin, TX, USA).

PAA modification with DNA. PAA membranes were washed sequentially with ethanol and ultrapure water to remove impurities on the surface and in the nanochannels, and then dried with nitrogen at room temperature. The modification process was shown in Figure S1. Amino groups were generated on the barrier layer of the PAA channel 
after gentle shaking in $5 \mathrm{~mL}$ of ethanol solution containing 5\% APTES for $12 \mathrm{~h}$. The PAA membranes were then washed with ethanol again to remove residual silylating reagents, dried with nitrogen. Then, $20 \mu \mathrm{L}$ of $15 \mu \mathrm{M}$ Azo-DNA solution was added onto the surface of the PAA membrane and allowed to react for $24 \mathrm{~h}$. It was worth noting that the PAA membrane was hung in an airtight glass bottle with some water at the bottom. The saturated moisture in the bottle prevented the $20 \mu \mathrm{L}$ Azo-DNA solution from evaporating over $24 \mathrm{~h}$. PAA membrane was immersed in $1 \mathrm{~mL}$ of ultrapure water containing $0.1 \%$ benzaldehyde and shaken gently for $12 \mathrm{~h}$ to block the remaining amino groups and then the PAA membranes were washed with ultrapure water to remove the unbound DNA and residual benzaldehyde. Then, the functionalized PAA membrane with Azo-DNA immobilized on the barrier layer was obtained and stored in Tris buffer solution at $4{ }^{\circ} \mathrm{C}$.

Electrochemical measurements. A self-made electrochemical detection device was used. A PAA membrane $(15 \mathrm{~mm} \times 15 \mathrm{~mm})$ was placed in the middle of an $\mathrm{H}$-type Electrolytic cell. Two $\mathrm{Ag} / \mathrm{AgCl}$ electrodes were inserted into $1 \mathrm{mM} \mathrm{KCl}$ solution in the two chambers, one of which was used as the working electrode and the other as reference/counter electrode, respectively. CHI 660 D recorded the electrochemical linear sweep voltammetry from 1.0 to $+1.0 \mathrm{~V}$.

CD Spectroscopic Measurements. CD spectra were collected from 220 to $350 \mathrm{~nm}$ on a Chirascan circular spectropolarimeter (Applied Photophysis, England). The $15 \mu \mathrm{M}$ DNA samples were obtained by directly diluting the solid powder of DNAs in TE buffer ( $\mathrm{pH}$ 8.0). The lamp was kept under a stable stream of dry purified nitrogen (99.99\%) during experiments.

Optimization of Azo-DNA concentration. For the ion channel based on the interaction between GO and Azo-DNA-modified PAA channel, Azo-DNA concentration has played an important role in the switching ability. Figure S9A demonstrates the ion rectification behavior with the increasing Azo-DNA concentration from $2.5 \mu \mathrm{M}$ to 20 $\mu \mathrm{M}$. Moreover, the ionic current values of $-1.0 \mathrm{~V}$ versus Azo-DNA concentration are 
shown in Figure S9B. Similar to the GO concentration-characterized ion current curve, the slope decreases at high concentrations, which is the same reason that there is no space to accommodate, so $15 \mu \mathrm{M}$ was chosen in the further exploration.

Optimization of GO size. As an important element of the ionic gate, GO blocks the channel to control the states of the gate. Three different sizes of GO, $<0.5 \mu \mathrm{m}, 0.5 \mu \mathrm{m}$ $5 \mu \mathrm{m}$, and $>5 \mu \mathrm{m}$, have been adopted to explore the effect of GO size on the performance of this gate. As shown in Figure S10, compared with the other two sizes, GO with the size of $0.5 \mu \mathrm{m}-5 \mu \mathrm{m}$ shows best gating efficiency.

Stability of the DNA attachment to the surface. To verify the stability of the imine attachment of the DNA to the surface in water, experiments have also been conducted. After being immersed in water for $0 \mathrm{~h}, 12 \mathrm{~h}, 24 \mathrm{~h}$, and $36 \mathrm{~h}$, the immobilized Azo-DNA on the surface of PAA membrane was characterized by X-ray photoelectron spectroscopy (XPS). As shown in Figure S11, the peak of P 2p slowly decreases with the extension of immersion time, but enough DNA for the fabrication of ionic gate still exists. 
Table S1. XPS data of the bare PAA membrane.

\begin{tabular}{c|c|c|c|c|c|c|c}
\hline Name & Start BE & Peak BE & End BE & Height CPS & FWHM eV & Area (P) CPS.eV & Atomic \% \\
\hline C1s & 296.13 & 284.76 & 279.23 & 10289.32 & 1.84 & 29058.54 & 94.78 \\
\hline N1s & 410.13 & 400.09 & 392.23 & 806.39 & 1.62 & 2606.4 & 5.22 \\
\hline
\end{tabular}

Table S2. XPS data of the APTES-modified PAA membrane.

\begin{tabular}{c|c|c|c|c|c|c|c}
\hline Name & Start BE & Peak BE & End BE & Height CPS & FWHM eV & Area (P) CPS.eV & Atomic \% \\
\hline C1s & 296.48 & 284.78 & 279.58 & 13896.83 & 1.72 & 32198.8 & 86.72 \\
\hline N1s & 410.48 & 401.61 & 392.58 & 2063.86 & 2.57 & 8032.61 & 13.28 \\
\hline
\end{tabular}

Table S3. XPS data of the Azo-DNA-modified PAA membrane.

\begin{tabular}{c|c|c|c|c|c|c|c}
\hline Name & Start BE & Peak BE & End BE & Height CPS & FWHM eV & Area (P) CPS.eV & Atomic \% \\
\hline C1s & 296.58 & 284.79 & 279.68 & 10421.6 & 2.3 & 27118.81 & 81.77 \\
\hline N1s & 410.58 & 400.76 & 392.68 & 2409.79 & 3.84 & 9848.01 & 18.23 \\
\hline
\end{tabular}

Table S4. XPS data of the Azo-DNA-modified PAA membrane treated with GO.

\begin{tabular}{c|c|c|c|c|c|c|c}
\hline Name & Start BE & Peak BE & End BE & Height CPS & FWHM eV & Area (P) CPS.eV & Atomic \% \\
\hline C1s & 296.58 & 284.78 & 279.68 & 15290.17 & 1.87 & 36271.98 & 83.81 \\
\hline N1s & 410.58 & 401.11 & 392.68 & 2951.21 & 2.35 & 11415.08 & 16.19 \\
\hline
\end{tabular}



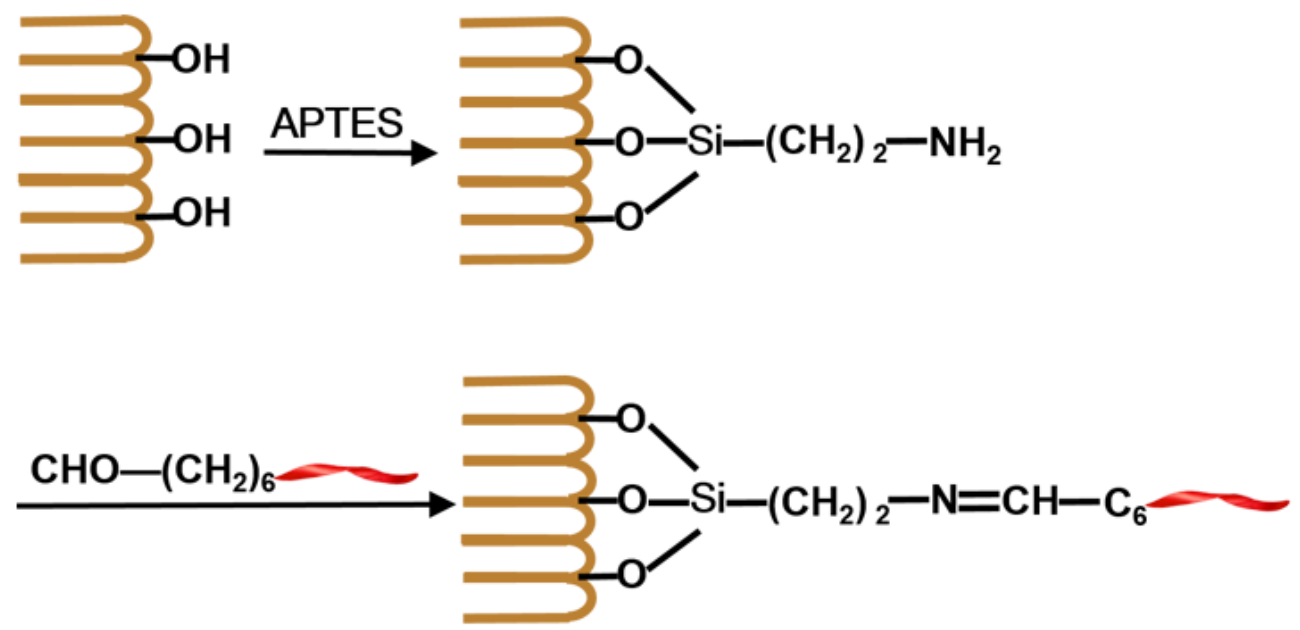

Figure S1. Schematic of the surface modification process of the Azo-DNA on the barrier layer of PAA.

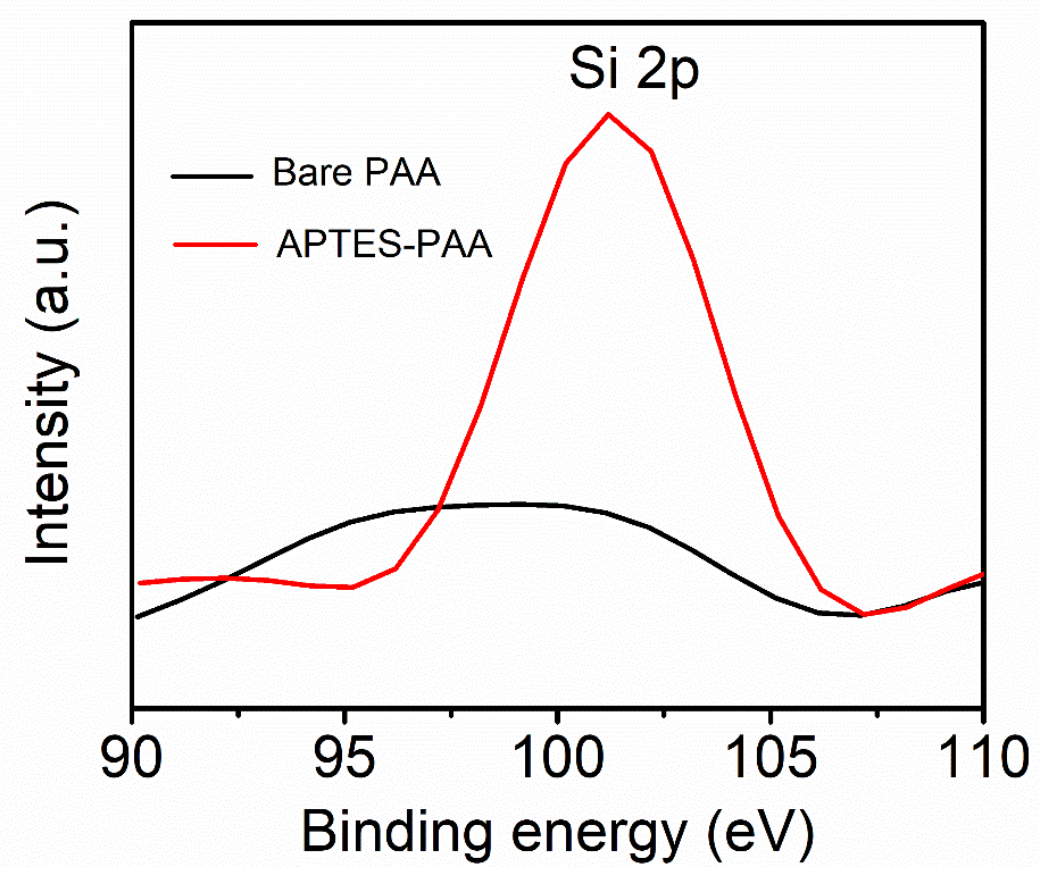

Figure S2. XPS spectra of bare PAA membrane and PAA membrane with $\mathrm{NH}_{2}$ activated focusing on $\mathrm{Si}$. 


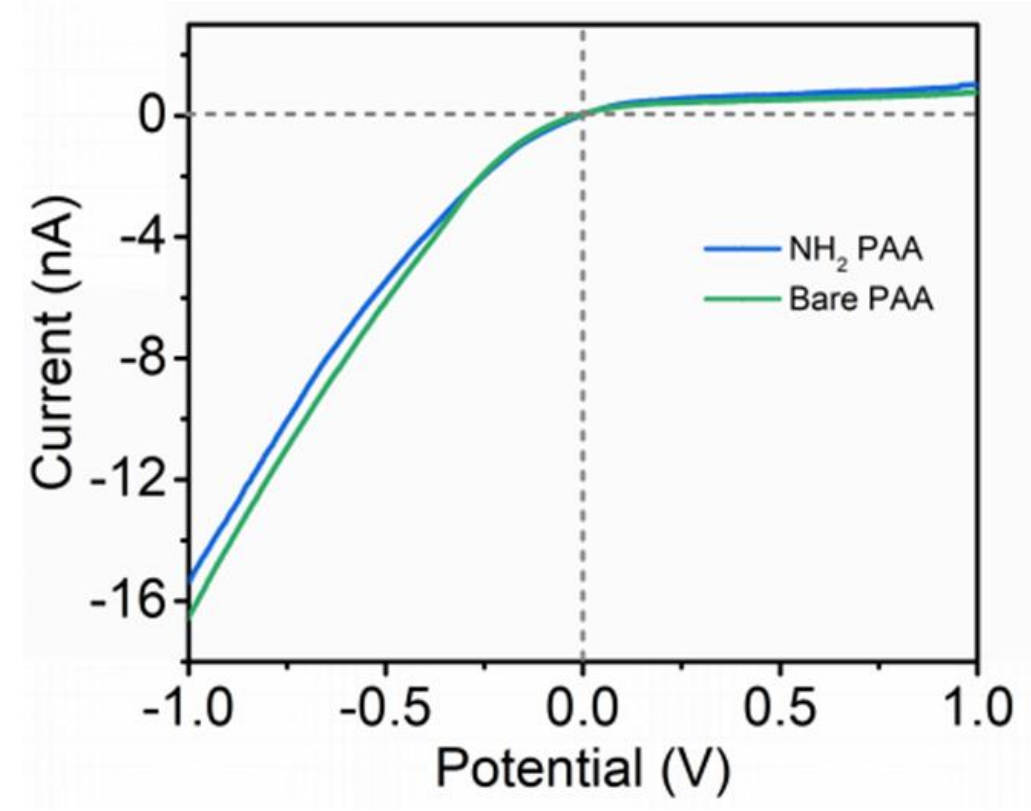

Figure S3. $I-V$ properties of the bare PAA membrane and PAA membrane with $\mathrm{NH}_{2}$ activated.

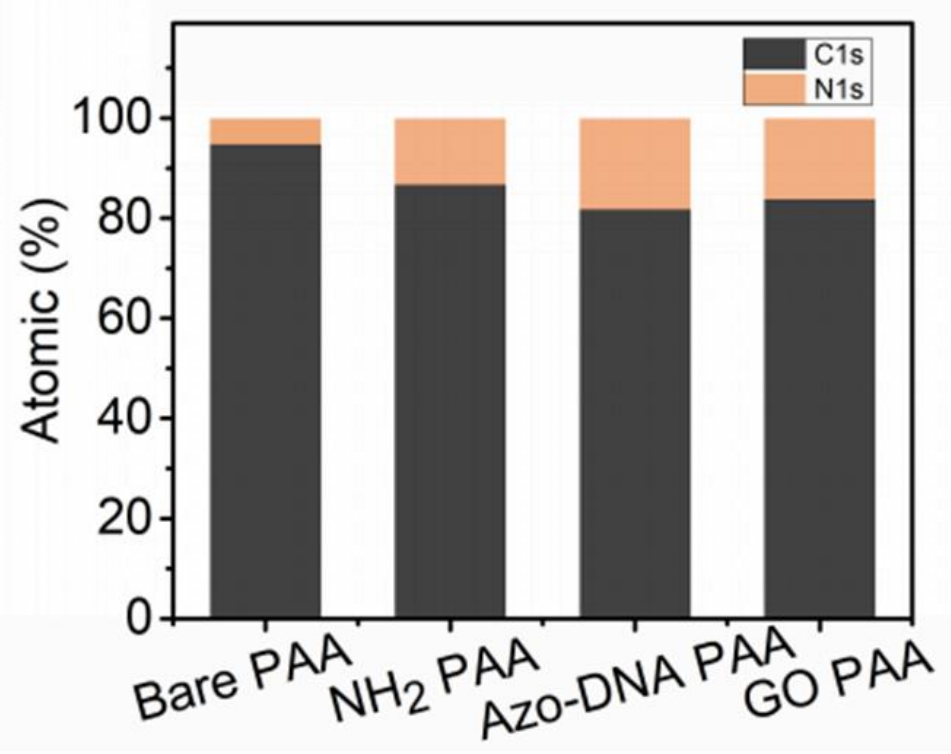

Figure S4. Ratio of $\mathrm{C}$ and $\mathrm{N}$ of the PAA channel membrane, modified with APTES, Azo-DNA, and GO. 


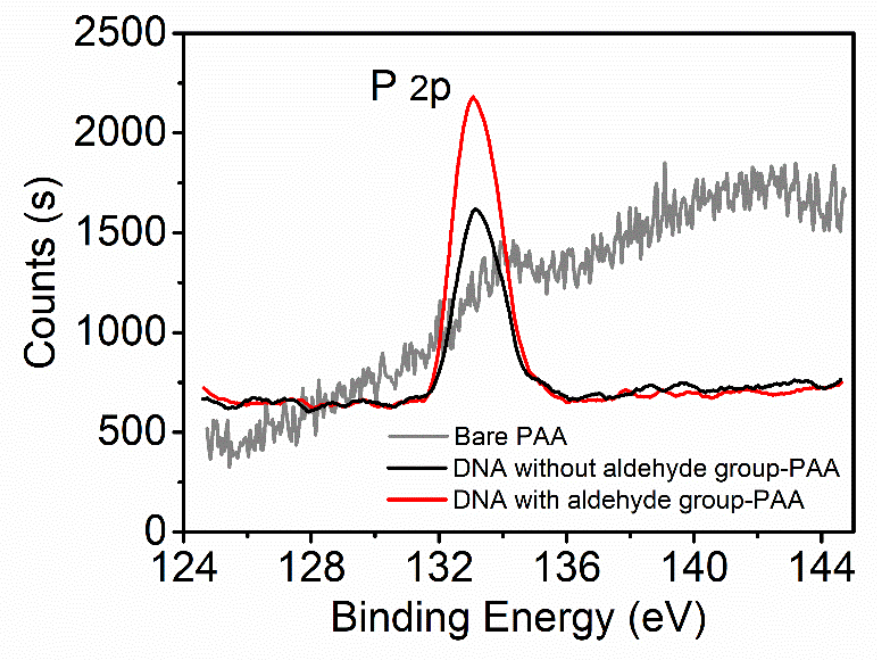

Figure S5. XPS spectra of bare PAA, PAA modified with Azo-DNA with aldehyde group, and PAA modified with Azo-DNA without aldehyde group focusing on P.

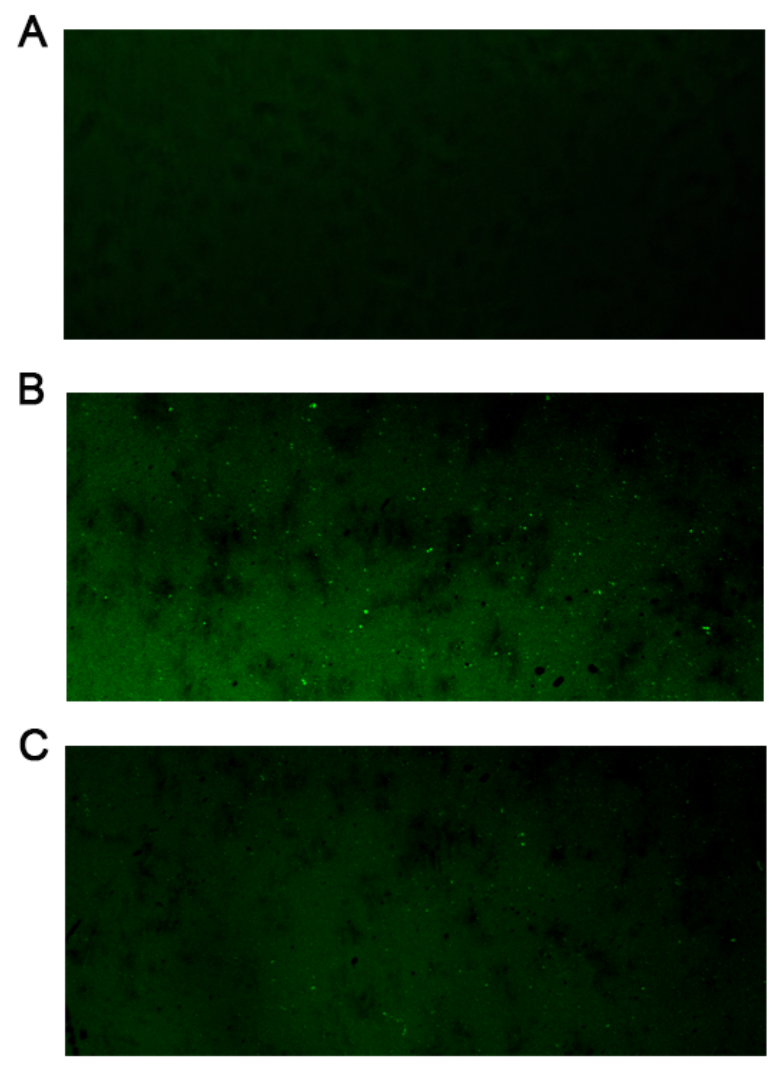

Figure S6. LSCM characterizations of the Azo-DNA on the surface of (A) bare PAA, (B) PAA modified with Azo-DNA with aldehyde group, and (C) PAA modified with Azo-DNA without aldehyde group. 


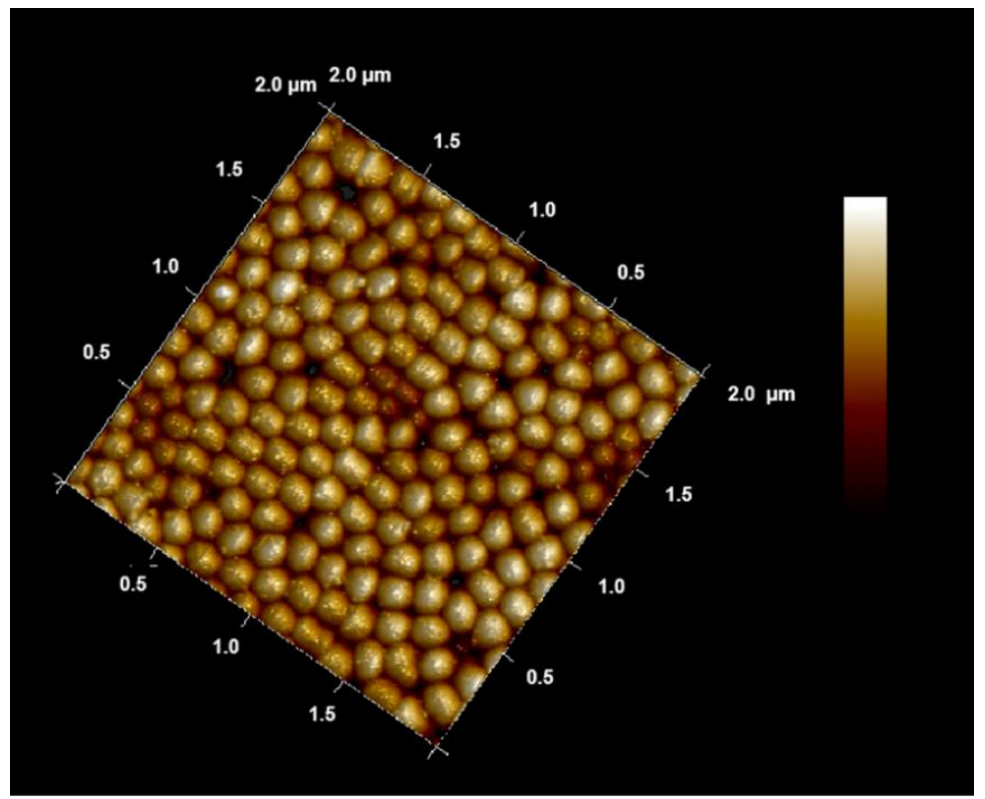

Figure S7. AFM image of PAA membrane view from the barrier layer side.
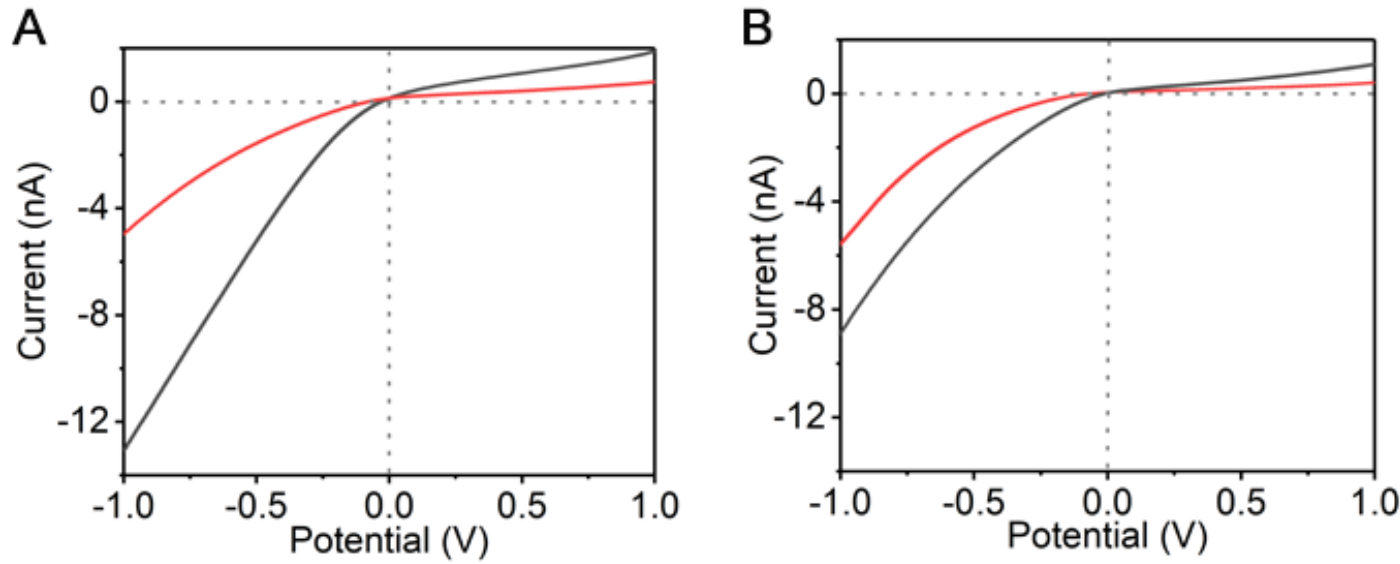

Figure S8. $I$ - $V$ properties of the ion channel in PBS solution (A) and blood plasma (B). (Grey curve: under irradiation of visible light; Red curve: under irradiation of ultraviolet light). 

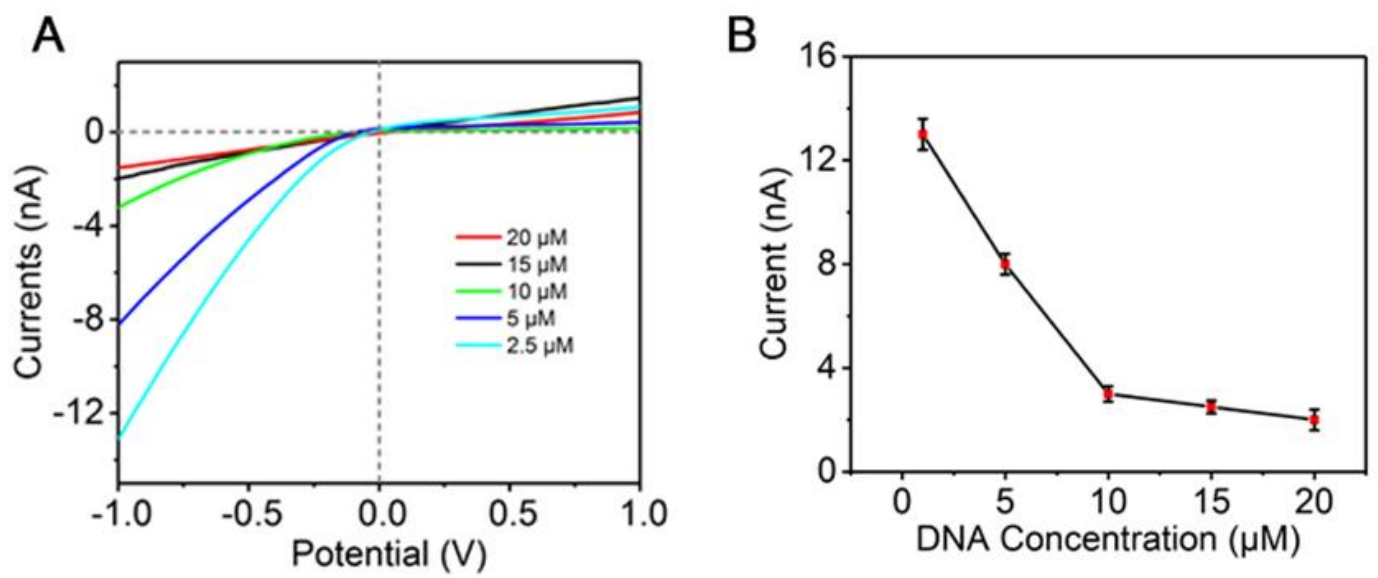

Figure S9. Effect of Azo-DNA concentration on ion transport. (A) $I-V$ properties of the ion channel with different concentrations of Azo-DNA. (B) The ionic current values at $-1.0 \mathrm{~V}$ vs Azo-DNA concentration.
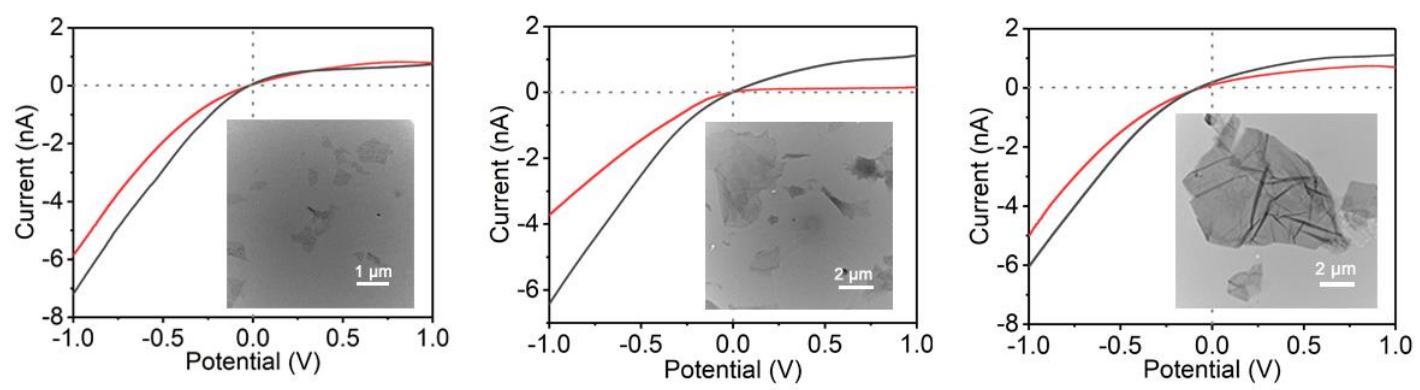

Figure S10. $I-V$ properties of the ion channel with different diameters of GO. (Grey curve: under irradiation of visible light; Red curve: under irradiation of ultraviolet light). 


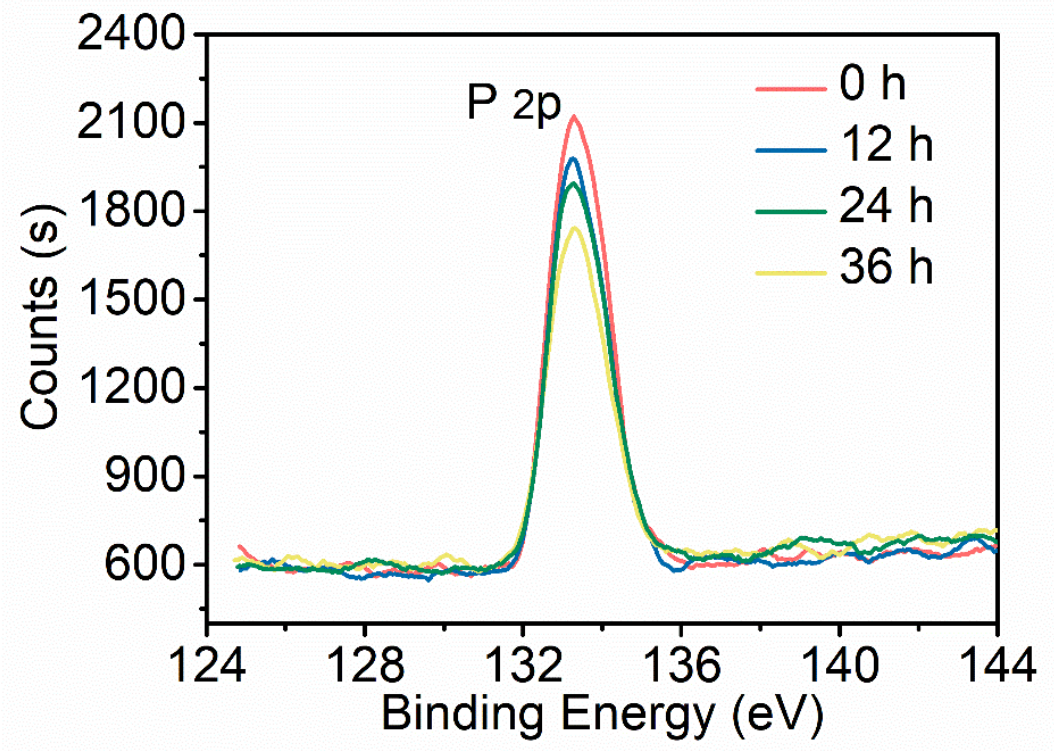

Figure S11. XPS spectra of Azo-DNA-modified PAA after immersion in water for $0 \mathrm{~h}$, $12 \mathrm{~h}, 24 \mathrm{~h}$, and $36 \mathrm{~h}$ focusing on $\mathrm{P}$. 